\title{
ESCALA DE BRADEN: BENEFÍCIOS DE SUA APLICAÇÃO NA PREVENÇÃO DE LESÃO POR PRESSÃO NO ÂMBITO DOMICILIAR
}

\author{
Nataline Rocha de Lima ${ }^{1}$ \\ Nataly Rocha de Lima \\ Joquebede Costa de Oliveira Souza ${ }^{3}$ \\ Taiana da Silva Silvério ${ }^{4}$ \\ José Ossian Almeida Souza Filho \\ Tiago dos Santos-Nascimento ${ }^{6}$
}

LIMA, N. R. de; LIMA, N. R. de; SOUZA, J. C. de O.; SILVÉRIO, T. da S.; SOUZA FILHO, J. O. A.; SANTOS-NASCIMENTO, T. D. Escala de Braden: benefícios de sua aplicação na prevenção de lesão por pressão no âmbito domiciliar. Arquivos de Ciências da Saúde da UNIPAR, Umuarama, v. 25, n. 2, p, 95-103, maio/ago. 2021.

RESUMO: Objetivo: Averiguar a eficácia da Escala de Braden como instrumento norteador na assistência para prevenção de lesão por pressão em indivíduos acamados no âmbito domiciliar. Método: Trata-se de um estudo descritivo com abordagem quantitativa com coleta de dados realizada em domicílio por meio de entrevista e inspeção da pele de 41 participantes. Resultados: A incidência de lesão por pressão foi de 7\%. Constatou-se que os indivíduos acamados em domicílio com alto risco, na escala de Braden, não mostraram incidência de lesão por pressão, enquanto os que apresentaram risco moderado houve incidência. Conclusão: Apesar de ser um instrumento bastante utilizado pelos profissionais da área da saúde, o presente trabalho demonstrou que a alta sensibilidade e especificidade da Escala de Braden é questionável. Os cuidados foram essênciais para essa baixa incidência de lesão por pressão, diminuindo sua associação com a idade e tempo de acamado.

PALAVRAS-CHAVE: Lesão por Pressão. Cuidados de Enfermagem. Atenção Primária à Saúde. Escala Braden.

\section{BRADEN SCALE: BENEFITS OF ITS USE IN THE PREVENTION OF PRESSURE INJURY IN THE HOUSEHOLD}

ABSTRACT: Objective: investigate the effectiveness of the Braden Scale as a guiding instrument in assisting in the prevention of pressure injury in bedridden individuals at home. Method: This is a descriptive study with a quantitative approach, with data collected at home through interviews and skin inspection of 41 participants. Results: There was an incidence of $7 \%$ of pressure injuries. It was found that individuals at high risk at home, according to the Braden scale, did not show incidence of pressure injuries, while those with moderate risk presented such incidence. Conclusion: Despite being widely used by health professionals, this study demonstrated that the high sensitivity and specificity of the Braden Scale is questionable. Care was essential for this low incidence of pressure injuries, reducing its association with age and bedridden time.

KEYWORDS: Pressure Injury. Nursing care. Primary Health Care. Braden Scale.

\section{Introdução}

A lesão por pressão (LP) consiste em um dano que ocorre na pele e/ou nos tecidos subjacentes, geralmente sobre uma proeminência óssea. Pode se apresentar como pele íntegra (eritema não branqueável) ou como úlcera. É resultante de pressão prolongada e/ou intensa em combinação com o cisalhamento, e também pode estar relacionada ao uso de dispositivo médico (COREN, 2016). A LP ainda se configura como um grave problema de saúde pública devido sua elevada incidência e prevalência tanto no âmbito hospitalar como no domiciliar, apesar de ser uma lesão prevenível (CAMPOS et al., 2016), fazendo-se necessária a adoção na prática clínica de instrumentos que norteiem a assistência na prevenção desse agravo à saúde (BRASIL, 2013).

As taxas de incidência e prevalência de LP são variadas quando diagnosticadas por meio da aplicação do Protocolo para Prevenção de Úlcera por Pressão, do Programa Nacional de Segurança do Paciente (PNSP), do Ministério da Saúde (MS), diferenciando-se conforme o nível de cuidado (cuidados de longa permanência, cuidados agudos e cuidados domiciliares). No que tange a atenção domiciliar as taxas de prevalência e incidência atingem até $29 \%$ e 17\%, respectivamente (BRASIL, 2013). Pesquisas realizadas em estados do Nordeste e Sudeste apresentaram incidência variando entre 20 a 30\% (VIEIRA et al., 2016; MORO; CALARI, 2016).

A utilização de escalas de predição, como a Escala de Braden (EB), é comumente usada como parte de instrumentos metodológicos e diretrizes/protocolos clínicos para a prevenção de LP (DEBON et al., 2018; VIEIRA et al., 2018; ALVES; COSTA; BOUÇÃO, 2016). Assim, a EB subsidia a avaliação clínica realizada pelo enfermeiro, bem como norteia o julgamento clínico e a tomada de decisões, auxiliando assim na construção de um plano de cuidados individualizado para identificar e impedir que pacientes desenvolvam LP, norteando assim a escolha de medidas preventivas apropriadas e efetivas para cada paciente (ALVES; COSTA; BOUÇÃO, 2016; DEBON et al., 2018).

Contudo, percebe-se que a maioria dos hospitais não institucionaliza a utilização de protocolos ou escalas

DOI: https://doi.org/10.25110/arqsaude.v25i2.2021.7995

${ }^{1}$ Discente do Curso de Bacharelado em Enfermagem pela Faculdade do Vale do Jaguaribe-FVJ, e-mail: natalineroch93@hotmail.com ${ }^{2}$ Discente do Curso de Bacharelado em Enfermagem pela Faculdade do Vale do Jaguaribe-FVJ, e-mail: natalylima20@outlook.com ${ }^{3}$ Discente do Curso de Bacharelado em Enfermagem pela Faculdade do Vale do Jaguaribe-FVJ, e-mail: jokede23oliveira@gmail.com ${ }^{4}$ Discente do Curso de Bacharelado em Enfermagem pela Faculdade do Vale do Jaguaribe-FVJ, e-mail: silveriotayana@gmail.com ${ }^{5}$ Biólogo. Mestre em Bioquímica pela Universidade Federal do Ceará-UFC. Docente da faculdade do vale do Jaguaribe, e-mail: ossian@fvj.br ${ }^{6}$ Biólogo, Doutor em Biotecnologia-RENORBIO, pela Universidade Estadual do Ceará-UECE. Docente da Faculdade do Vale do Jaguaribe-FVJ, e-mail: santosnascimento.t@gmail.com 
para prevenção de LP. Esse cenário também perfaz a atenção domiciliar, onde a maioria dos cuidados prestados na prevenção e tratamento é executada pelo cuidador ou familiar, que fazem de forma empírica, sem orientação e supervisão de um profissional de saúde (VIEIRA et al., 2016).

Assim, surgem os seguintes questionamentos: Quais os benefícios da utilização da EB no âmbito domiciliar na prevenção de LP? Quais os indivíduos acamados que apresentam maior risco de desenvolver LP? Quais os cuidados adotados para prevenção da LP?

Diante do exposto, é possível que a EB seja um instrumento de grande valia para subsidiar a assistência de enfermagem no âmbito domiciliar no que se refere à prevenção de LP. Assim o presente estudo tem como objetivo averiguar a eficácia da Escala de Braden como instrumento norteador na assistência para prevenção de lesão por pressão em indivíduos acamados no âmbito domiciliar.

\section{Material e Método}

Trata-se de um estudo descritivo com abordagem quantitativa. De acordo com Cervo, Bervian e Silva (2014), a pesquisa descritiva objetiva observar, registrar, analisar e correlacionar fatos e fenômenos da realidade sem haver interferência sobre eles. A pesquisa quantitativa quantifica opiniões, problemas e informações, partindo da premissa que o entendimento é facilitado a partir dos números, para isso também se faz uso de técnicas estatísticas (MICHEL, 2009).

Este estudo foi desenvolvido nas regiões adscritas à Estratégia Saúde da Família (ESF) de nove Unidades Básicas de Saúde (UBS), situadas na zona urbana do município de Aracati, Ceará, com indivíduos de ambos os sexos, maiores de 18 anos, acamados em domicílio, que puderam responder o formulário ou dispuseram de um cuidador para fazer isso.

A localização e identificação dos participantes foram realizadas a partir do banco de dados da Secretaria Municipal de Saúde, onde consta um total de 136 indivíduos acamados em domicílio, contudo 19 endereços estavam incompletos e nove endereços não foram localizados via Google Maps (Google, Estados Unidos), resultando assim, em um quantitativo de 108 participantes.

Entretanto, a amostra representativa determinada por cálculo amostral, foi composta por 85 participantes, escolhidos aleatoriamente. Estes foram agrupados em três grupos: menores de 60 anos (NI - não idoso), entre 60 e 76 anos (I - idoso) e maiores de 76 anos (AE - idoso acima da expectativa de vida). $\mathrm{O}$ agrupamento foi realizado mediante os seguintes critérios: segundo o Estatuto do Idoso, pautado na Lei $\mathrm{n}^{\circ} 10.741 / 03$, o idoso é o indivíduo com idade igual ou superior a 60 anos. Assim os menores de 60 anos não são idosos (NI) e o segundo grupo etário compreende a população considerada idosa, se estendendo até a expectativa de vida do brasileiro, que de acordo com o Instituto Brasileiro de Geografia e Estatística (IBGE) (2018), é de 76 anos. O último grupo foi constituído por aqueles participantes cuja faixa etária superou a referida expectativa de vida.

Antes de iniciar a pesquisa cada enfermeiro de sua respectiva UBS foi informado sobre o estudo que seria realizado. A participação foi condicionada ao prévio consentimento do participante ou de seu responsável e a assinatura do Termo de Consentimento Livre e Esclarecido, com as adequadas orientações e esclarecimentos sobre a pesquisa.

A coleta de dados ocorreu durante o mês de outubro de 2019, através de um formulário aplicado pelos pesquisadores pessoalmente aos participantes na presença dos cuidadores, onde o exame físico da pele também foi realizado. O formulário utilizado foi composto por cinco partes. $1^{\circ}$ ) característica sociodemográficas; $2^{\circ}$ ) dados clínicos do paciente; $3^{\circ}$ ) condições da pele; $4^{\circ}$ ) risco para desenvolvimento de lesão por pressão utilizando a EB; $5^{\circ}$ ) medidas preventivas utilizadas pelo cuidador.

O instrumento utilizado para avaliar o risco de lesão por pressão foi a Escala de Braden, validada para o Brasil por Paranhos e Santos (1999). Essa escala é composta por seis subescalas, sendo que 03 mensuram a intensidade e duração da pressão - percepção sensorial, atividade e mobilidade; e 03 determinam a tolerância da pele e estruturas de sustentação à pressão - umidade, nutrição, fricção e cisalhamento (BRASIL, 2013).

As cinco primeiras subescalas, percepção sensorial, umidade, atividade, mobilidade e nutrição, são pontuadas de 1 (menos favorável) a 4 (mais favorável), e a sexta subescala, fricção e cisalhamento, é pontuada de 1 a 3. Cada subescala é composta por um título com definição, e cada nível apresenta uma categoria com sua respectiva descrição. Essa escala apresenta um escore total de 6 a 23 pontos, sendo que quando menor o escore maior o risco para desenvolvimento de lesão por pressão (BRASIL, 2013).

O Protocolo para Prevenção de Úlcera por Pressão recomenda que o risco de desenvolvimento de LP seja categorizado em cinco níveis pelo uso da EB: baixo risco pacientes com escore de 15 a 18; risco moderado - pacientes com escore de 13 a 14; risco alto - pacientes com escore de 10 a 12; e risco muito alto - pacientes com escore igual ou menor que 9 (BRASIL, 2013).

Todos os dados foram expressos como média \pm E.P.M. (erro padrão da média), em que ' $n$ ' indica o número de indivíduos entrevistados. O teste One Way Anova não pareado foi utilizado quando apropriado. Considerou-se $\mathrm{P}$ $<0,05$ para indicar a diferença estatisticamente significante. Foi utilizando o Graphprism 8.3 (GraphPad Software, San Diego, EUA) para as análises estatísticas.

$\mathrm{O}$ estudo segue as recomendações éticas da Resolução no 466/12 do Conselho Nacional de Saúde e foi aprovado pelo Comitê de Ética em Pesquisa da Faculdade do Vale do Jaguaribe sob protocolo $n^{\circ}$ 18410519.3.0000.9431.

\section{Resultados e Discussões}

Na fase de visitação para entrevistas, 09 se recusaram a participar da pesquisa, 09 não tiveram seus endereços encontrados, 23 tinham falecidos, 02 estavam hospitalizados e 01 mudou de endereço. Assim, dos 85 indivíduos acamados em domicílio selecionados inicialmente, 41 participaram da pesquisa. 


\section{Dados Sociodemográficos}

A população investigada se concentrou no grupo I e AE (Figura 1), dessa forma a distribuição de indivíduos por grupo é o inverso daquela apresentada pela pirâmide populacional (IBGE, 2018). Contudo, o encontrado atendeu as expectativas, uma vez que, foi investigado um grupo especial de indivíduos acamados, condição que está relacionada positivamente com o avanço da idade.

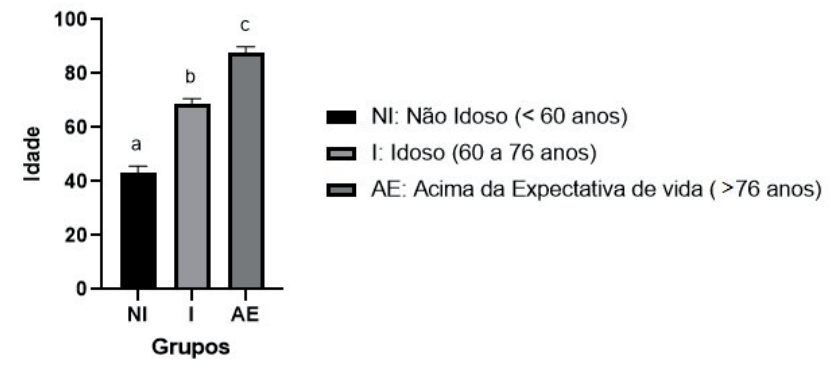

Figura 1: Média de idade dos grupos

O gráfico 1 mostra a relação da média de idade dos grupos, sendo que o grupo NI (não idoso) corresponde aos participantes menores de 60 anos, o grupo I (idoso) corresponde aos participantes entre 60 a 76 anos e o grupo AE (acima da expectativa de vida) compreende os maiores de 76 anos.

a - O grupo NI mostrou significância em relação ao I e AE (One Way Anova, $\mathrm{P}<0,001$ ).

b - O grupo I mostrou significância entre o grupo NI e AE (One Way Anova, $\mathrm{P}<0,001$ )

c - O grupo AE mostrou significância em relação ao grupo NI e I (One Way Anova, $\mathrm{P}<0,001$ ).

Fonte: Dados da pesquisa (2019).

Na amostra houve predominância do sexo masculino (Tabela 1), o que diverge com os dados da Pesquisa Nacional por Amostra de Domicílios Contínua (PNAD Contínua) (2018). Segundo a qual o número de mulheres no Brasil é superior ao de homens, correspondendo o público feminino a $51,7 \%$ e o público masculino a $48,3 \%$. Essa divergência pode ter ocorrido pelo fato da amostra ter sido escolhida a partir de um grupo especial não considerado nas pesquisas do IBGE. Contudo, quanto à etnia, os dados da presente pesquisa (Tabela 01) convergem com a PNAD Contínua (2018), onde mostra que $46,5 \%$ dos brasileiros se declaram como pardos, $43,1 \%$ como brancos e $9,3 \%$ como negros.

Tabela 1: Dados sociodemográficos dos participantes.

\begin{tabular}{llccc}
\hline \multicolumn{1}{c}{ Variável } & Descrição & NI & I & AE \\
\hline \multirow{2}{*}{ Sexo (\%) } & Masculino & 88,89 & 58,33 & 25 \\
& Feminino & 11,11 & 41,61 & 75 \\
& Brancos & 22,22 & 16,67 & 55 \\
Cor (\%) & Pardos & 66,67 & 66,67 & 30 \\
& Negros & 11,11 & 16,67 & 15 \\
& Solteiro & 66,67 & 33,33 & 10 \\
Estado Civil & Casado & 11,11 & 58,33 & 15 \\
(\%) & Divorciado & 22,22 & 8,33 & 5 \\
& Viúvo & 0 & 0 & 70
\end{tabular}




\section{Condições Clínicas}

Ao que se refere ao tempo de acamamento houve uma diferença significativa entre o grupo NI e os grupos I e $\mathrm{AE}$ (One Way Anova, $\mathrm{P}<0,05$ ) visto que o primeiro grupo expressou um tempo médio de $25,18 \pm 5,81$ anos, enquanto o grupo I apresentou um tempo médio de $5,81 \pm 1,48$ anos e o grupo AE de 4,95 \pm 1,14 anos (Gráfico 2).

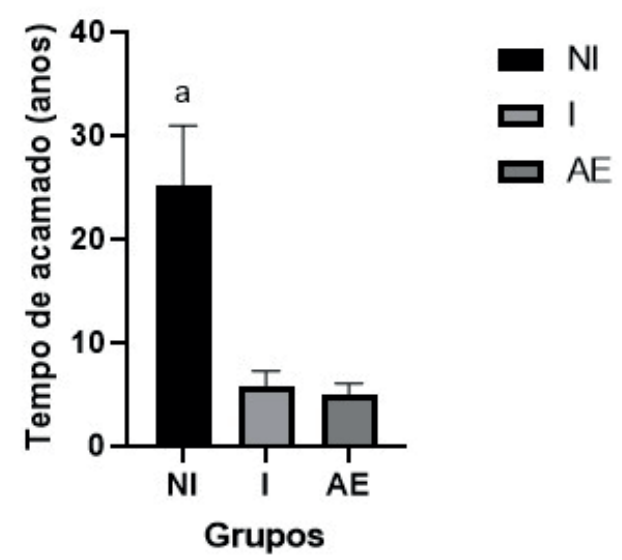

Figura 2: Tempo de acamamento dos participantes da pesquisa.

O gráfico 2 mostra a relação do tempo de acamamento com as idades dos participantes, onde somente o grupo NI mostrou significância em relação aos demais grupos (a, One Way Anova, $\mathrm{P}<0,05$ ).

Fonte: Dados da pesquisa (2019).

Esse maior tempo de acamamento apresentado pelo grupo NI está diretamente relacionado à causa do acamamento, que foram as doenças neuronais, com ênfase para a paralisia cerebral. O tempo de acamamento entre os grupos I e AE, foi relativamente curto (Tabela 02), e isso está relacionado ao fato que o principal motivo dessa condição foi o acidente vascular encefálico (AVE), patologia que diminui a sobrevida e tem aumento de probabilidade com o avanço da idade (BERNARDES et al., 2019). Doenças que comprometem o sistema neurológico como o AVE, a esclerose múltipla e a doença de Alzheimer podem alterar a percepção sensorial, a atividade e a mobilidade, impactando dessa forma na capacidade de reagir ao excesso de pressão (CAMPOS et al., 2016).

Tabela 2: Dados clínicos dos participantes.

\begin{tabular}{llccc}
\hline Variável & Descrição & NI & I & AE \\
\hline \multirow{4}{*}{ Motivo (\%) } & Não sabe & 10 & 0 & 10 \\
& Vascular & 10 & 61,54 & 35 \\
& Neuronal & 70 & 30,77 & 25 \\
& Óssseo & 10 & 7,68 & 25 \\
& Outros & 0 & 0 & 5 \\
& Não & 36,36 & 9 & 7,41 \\
Comorbidades & Vascular & 27,27 & 36 & 62,96 \\
& Neuronal & 27,27 & 5 & 14,81 \\
& Metabólica & 0 & 45 & 14,81 \\
& Outras & 9,09 & 5 & 0
\end{tabular}

\begin{tabular}{llccc}
$\begin{array}{l}\text { Uso de } \\
\text { Medicações }\end{array}$ & Sim & 88,89 & 100 & 100 \\
$(\%)$ & Não & 11,11 & 0 & 0 \\
& Outras & 6,67 & 15 & 10,42 \\
\multirow{2}{*}{$\begin{array}{l}\text { Medicamentos } \\
(\%)\end{array}$} & Vascular & 20 & 32,5 & 37,5 \\
& Neuronal & 73,33 & 27,5 & 45,83 \\
& Metabólica & 0 & 25 & 6,25 \\
\hline Fonte: Dados da pesquisa (2019). & & &
\end{tabular}

No que concerne as comorbidades, o grupo NI revelou maior índice de pessoas sem comorbidades (Tabela 02). Dentre as doenças que esse grupo apresentou, destacase a hipotensão, a hipertensão arterial e a epilepsia. O grupo I apresentou maior índice de doenças metabólicas seguida pelas doenças vasculares, com destaque para dislipidemia, diabetes mellitus tipo II e hipertensão arterial. E o grupo AE apresentou maior percentual de comorbidades vasculares, com ênfase para hipertensão arterial, hipotensão e insuficiência cardíaca congestiva, além de outras doenças como depressão, ansiedade, epilepsia e doença de Parkison.

$\mathrm{O}$ uso de medicações contínuas foi relatado pela maioria dos participantes de todos os grupos, isso corrobora com o estudo de Vieira et al. (2016), onde o uso de medicações contínuas foi relatado por 90,6\% dos indivíduos acamados. No grupo NI houve predominância dos medicamentos neuronais, como os anticonvulsivantes, os sedativos, os antipsicóticos e os medicamentos para o tratamento de Alzheimer (Tabela 02). O grupo I houve prevalência das medicações vasculares, com destaque para os anti-hipertensivos. O grupo AE também apresentou maior uso de medicamentos neuronais, entre os quais estão os antidepressivos, os antipsicóticos, os sedativos, as drogas antiparkisônicas, os anticonvulsivantes e os hipnóticos.

Alguns medicamentos interferem sobre o nível de consciência, como os sedativos, os antipsicóticos e os analgésicos narcóticos, impactando dessa forma na percepação sensorial sobre o desconforto ocasionado pela pressão, esse fato tem influência direta no escore obtido pela EB (CAMPOS et al., 2016). Além disso, as drogas anti-hipertensivas predispõe o desenvolvimento de uma lesão, pois reduzem o fluxo sanguíneo e a perfusão tecidual (VIEIRA et al., 2016).

\section{Condições da Pele}

A etiologia da LP é multicausal, envolvendo fatores intrínsecos e extrínsecos. Os fatores intrínsecos modificam a integridade e a constituição da pele e de suas estruturas internas tornando assim a pele menos resistente à pressão, além de influenciar no processo de cicatrização. Esses fatores incluem: nível de consciência, idade avançada, estado nutricional, desidratação, peso corporal (menos gordura, menos proteção sobre as proezas ósseas; já a obesidade dificulta a mobilização devido ao excesso de peso), mobilidade reduzida ou ausente, umidade, comorbidades, uso de medicamentos, anemia e tabagismo (CAMPOS et al., 2016; VIEIRA, et al., 2016; VIEIRA, et al., 2018). Como fatores extrínsecos, tem-se a pressão, umidade, temperatura, cisalhamento e fricção (ALVES; COSTA; BOUÇÃO, 2016; 
CAMPOS et al., 2016).

A incidência de lesão por pressão foi de $7 \%$ em domicílio e $2 \%$ devido hospitalização, sendo que apenas quatro dos 41 participantes desenvolveram LP. Com isso vêse que a incidência de LP foi baixa comparada com um estudo realizado com indivíduos acamados atendidos por unidades básicas em Teresina, Piauí, onde a incidência foi de 28,1\%, uma vez que nove dos 32 participantes apresentaram LP (VIEIRA et al., 2016). Este fato justifica-se por essa pesquisa ter sido realizada com uma amostra onde houve prevalência de idosos, cuja idade média era de 82,8 $\pm 14,0$ anos. Além disso, 59,4\% apresentavam pele ressecada e $81,2 \%$ turgor cutâneo diminuído. A incidência baixa de LP evidenciada nesse trabalho pode estar relacionada às medidas preventivas utilizadas pelos cuidadores.

As condições da pele interferem no surgimento de uma LP. A pele hidratada e com turgor cutâneo preservado assegura maior resistência a agentes externos, como pressão, fricção e cisalhamento, minimizando dessa forma sua ruptura (BRASIL, 2013). O grupo NI mostrou em sua totalidade um turgor cutâneo preservado e com um índice significativo de pele hidratada, resultando dessa forma em um índice zero para o desenvolvimento de LP, apesar de ser o grupo com maior tempo de acamamento (Tabela 03).

Já o grupo I e AE tiveram incidência de LP, apesar de terem menor tempo de acamamento (Tabela 03, Gráfico 02). Isso pode estar relacionado ao fato que esses grupos eram constituídos por idosos, população mais suscetível a traumas mecânicos e ao desenvolvimento de uma LP, principalmente naqueles que portam doenças crônicas e degenerativas (CAMPOS et al., 2016; DEBON et al., 2018; POTTER et al., 2013; VIEIRA, et al., 2016).

Tabela 3: Condições da pele.

\begin{tabular}{llccc}
\hline \multicolumn{1}{c}{ Variável } & Descrição & NI & I & AE \\
\hline Tipo de pela & Hidratada & 77,78 & 66,67 & 70 \\
(\%) & Ressecada & 22,22 & 33,33 & 30 \\
Turgor & Preservado & 100 & 91,67 & 50 \\
cutâneo (\%) & Diminuído & 0 & 8,33 & 50 \\
& Sim & 0 & 8,33 & 10 \\
Apresenta & Sim & 0 & 8,33 & 0 \\
lesão por & (hospital) & & & \\
pressão & Não & 100 & 83,33 & 90 \\
& Sacral & 0 & 100 & 0 \\
& Ombro & 0 & 0 & 33,33 \\
Local (\%) & Orelha & 0 & 0 & 33,33 \\
& Calcâneo & 0 & 0 & 33,33 \\
Já apresentou & Sim & 22,22 & 33,33 & 25 \\
lesão por & Sim & 11,11 & 25 & 5 \\
pressão & (hospital) & & & \\
& Não & 66,67 & 41,67 & 70 \\
& Sacral & 100 & 71,43 & 71,43 \\
Local & Trocanter & 0 & 28,57 & 14,29 \\
& Ombro & 0 & 0 & 14,29 \\
\hline
\end{tabular}

Fonte: Dados da pesquisa (2019).
Nessa etapa da vida a pele sofre diversas transformações próprias do processo de envelhecimento que corroboram para uma pele mais fina, susceptível à ruptura. Dentre as mudanças que ocorrem pode-se elencar a atrofia da derme que torna a pele mais fina e delicada; a redução da capacidade dos receptores sensoriais, havendo assim diminuição da percepção dos estímulos dolorosos; a redução da elasticidade e do turgor cutâneo; a diminuição do colágeno; a mudança na textura da pele, tornando-se mais seca devido à diminuição da atividade das glândulas sebáceas; a diminuição da vascularização; e a diminuição da massa muscular e do tecido subcutâneo (CAMPOS et al., 2016; DEBON et al., 2018; POTTER et al., 2013; VIEIRA, et al., 2016).

Quanto ao principal local de desenvolvimento da LP, a região sacral foi a mais acometida (Tabela 03), e isso converge com o estudo realizado em domicílio com pessoas acamadas onde esta região também foi a mais afetada, perfazendo 58,3\% (MORAES et al., 2019). Quando o indivíduo é posicionado em decúbito dorsal essa área sofre grande compressão, visto que é local de proeminência óssea, resultando assim em uma maior pressão sobre os tecidos e a pele, diminuindo o fluxo sanguíneo e consequentemente a oferta de oxigênio, o que pode culminar em injúria tissular (POTTER et al., 2013).

\section{Escore de risco para desenvolvimento de LP utilizando a EB}

Em relação à EB, na subescala percepção sensorial os grupos NI, I e AE apresentaram uma percepção sensorial com algum grau de limitação, na maioria dos participantes (Tabela 4). No grupo NI, esse fato pode estar associado à elavada incidência de doenças neuronais $(70 \%)$ e ao maior uso de medicações neuronais $(73,33 \%)$ que podem causar a diminuição da percepção sensorial. No grupo I e AE, pode estar relacionada ao AVE, patologia que pode resultar em sequelas neurológicas que diminuem a percepção sensorial (CAMPOS et al., 2016).

Tabela 4: Escala de Braden.

\begin{tabular}{|c|c|c|c|c|}
\hline Variável & Descrição & NI & I & $\mathbf{A E}$ \\
\hline \multirow{4}{*}{$\begin{array}{l}\text { Percepção } \\
\text { sensorial (\%) }\end{array}$} & $\begin{array}{l}\text { Completamente } \\
\text { limitado }\end{array}$ & 22,22 & 8,33 & 5 \\
\hline & Muito limitado & 22,22 & 16,67 & 15 \\
\hline & Pouco limitado & 55,56 & 50 & 50 \\
\hline & Sem limitações & 0 & 25 & 30 \\
\hline \multirow{4}{*}{ Umidade (\%) } & $\begin{array}{l}\text { Constantemente } \\
\text { úmido }\end{array}$ & 55,56 & 75 & 65 \\
\hline & Úmido & 0 & 8,33 & 5 \\
\hline & $\begin{array}{l}\text { Oacasionalmente } \\
\text { úmido }\end{array}$ & 11,11 & 8,33 & 10 \\
\hline & $\begin{array}{l}\text { Raramente } \\
\text { úmido }\end{array}$ & 33,33 & 8,33 & 20 \\
\hline \multirow{2}{*}{ Atividade (\%) } & Acamado & 33,33 & 16,67 & 20 \\
\hline & Cadeira & 33,33 & 75 & 40 \\
\hline
\end{tabular}




\begin{tabular}{|c|c|c|c|c|}
\hline \multirow{2}{*}{ Atividade (\%) } & $\begin{array}{l}\text { Anda } \\
\text { ocasionalmente }\end{array}$ & 33,33 & 8,33 & 40 \\
\hline & $\begin{array}{l}\text { Anda } \\
\text { frequentemente }\end{array}$ & 0 & 0 & 0 \\
\hline \multirow{4}{*}{$\begin{array}{l}\text { Mobilidade } \\
(\%)\end{array}$} & $\begin{array}{l}\text { Completamente } \\
\text { imóvel }\end{array}$ & 1,11 & 0 & 5 \\
\hline & Muito limitada & 33,33 & 50 & 20 \\
\hline & $\begin{array}{l}\text { Discretamente } \\
\text { limitada }\end{array}$ & 44,44 & 33,33 & 60 \\
\hline & Sem limitações & 11,11 & 16,67 & 15 \\
\hline \multirow{4}{*}{ Nutrição (\%) } & Muito precária & 0 & 8,33 & 5 \\
\hline & $\begin{array}{l}\text { Provavelmente } \\
\text { inadequada }\end{array}$ & 44,44 & 8,33 & 5 \\
\hline & Adequada & 11,11 & 75 & 50 \\
\hline & Excelente & 0 & 8,33 & 0 \\
\hline \multirow{3}{*}{$\begin{array}{l}\text { Friç̧ão e } \\
\text { Cisalhamento } \\
(\%)\end{array}$} & Problema & 77,78 & 58,33 & 40 \\
\hline & $\begin{array}{l}\text { Problema em } \\
\text { potencial }\end{array}$ & 22,22 & 41,67 & 40 \\
\hline & Sem problema & 0 & 0 & 20 \\
\hline
\end{tabular}

Fonte: Dados da pesquisa (2019).

$\mathrm{Na}$ subescala umidade, a maioria se apresentou constantemente úmido (Tabela 04). O contato frequente da pele com a umidade proviniente da transpiração, urina e fezes contribui para o aumento do risco de formação de uma lesão por pressão, visto que causa modificações no tegumento, diminuindo a resistência da pele a fatores físicos, como a pressão, a fricção e o cisalhamento. A umidade causa maceração da pele, e consequentemente sua ruptura (VIEIRA et al., 2016; CAMPOS et al., 2016; POTTER et al., 2013).

A atividade consiste no grau de atividade física realizada (POTTER et al., 2013). O grupo NI, I e AE apresentaram uma atividade prejudicada (Tabela 04). Isso está relacionado às comorbidades apresentadas por essa população que trouxeram sequelas motoras, impactando de forma negativa na locomoção.

Por outro lado, a mobilidade é a capacidade de mudar e controlar a posição do corpo (POTTER et al., 2013); e no grupo NI, a mobilidade apresentou-se preservada na maior parte dos casos, com isso vê-se que as doenças neurológicas não trouxeram tantos danos a mobilidade. Já os grupos I e AE, apresentaram certa limitação na mobilidade, e isso pode estar associada às sequelas deixadas pelo AVE e às doenças neurológicas.

A nutrição apresentou-se adequada na maioria dos participantes (Tabela 04). A resistência dos tecidos à pressão é influenciada pela nutrição. $\mathrm{O}$ aporte nutricional insuficiente torna a pele mais susceptível ao desenvolvimento de uma lesão, visto que a diminuição de peso e consequentemente de tecido subcutâneo resulta em contato frequente da pele com as proeminências ósseas, o que pode levar à ruptura. Além disso, está relacionada à debilidade geral que resulta em menor mobilidade, e assim maior tempo de pressão sobre as proezas ósseas (CAMPOS et al., 2016).

$\mathrm{Na}$ subescala fricção e cisalhamento a maioria apresentaram problema (Tabela 04), ou seja, na mobilização há atrito da pele com a superfície de repouso, além disso, frequentemente escorregam na cama ou na cadeira, necessitando de ajustes frequentes na posição (POTTER et al., 2013). A fricção está relacionada ao fato que somente uma pessoa realizava a mudança de decúbito e com isso os participantes eram arrastados no leito. Os indivíduos que apresentam mobilidade prejudicada não conseguem muitas vezes ajudar em sua mobilização, dificultando a elevação para a mudança de decúbito.

A friç̧ão consiste na força mecânica de duas superfícies movendo-se entre si, causando assim um dano tecidual superficial. A fricção ocorre quando a pele é arrastada por uma superfície, como no caso dos indivíduos movidos no leito sem elevação (CAMPOS et al., 2016; POTTER et al., 2013). O cisalhamento diferentemente da fricção acarreta em dano tecidual profundo, visto que consiste no deslizamento dos tecidos mais profundos (músculos e ossos) enquanto a pele e o tecido subcutâneo ficam estacionados (CAMPOS et al., 2016; POTTER et al., 2013). Isso ocorre quando os indivíduos permanecem sentados ou em decúbito dorsal com a cabeceira do leito elevada a mais de $30^{\circ}$, durante tempo prolongado (CAMPOS et al., 2016).

Os resultados após a aplicação da EB mostraram um escore médio de 12,89 $\pm 0,71$ para o grupo NI, 13,25 $\pm 0,94$ para o grupo I e 14,25 $\pm 1,19$ para o grupo AE (Gráfico 3). Apesar dos grupos serem bem distintos, não houve diferença estatisticamente significante, talvez pelo fato das condições clínicas se assemelharem.

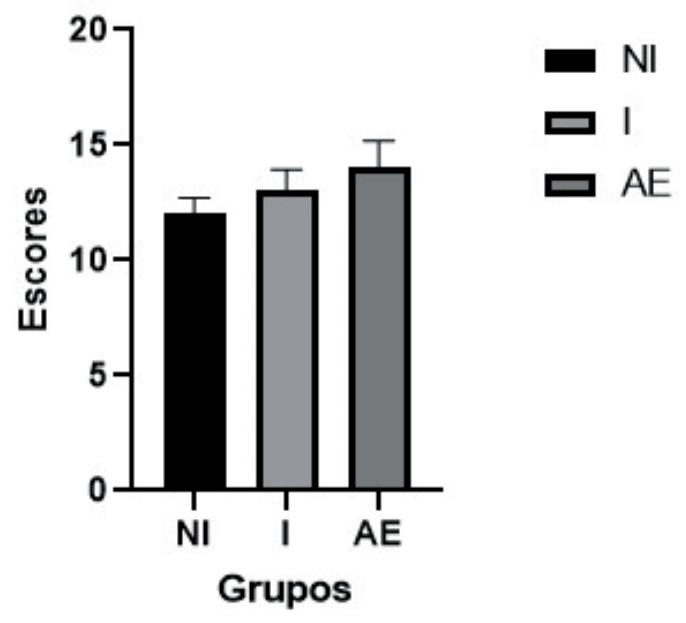

Figura 3: Média dos escores dos participantes da pesquisa Este gráfico mostra a média dos escores da escala de Braden dos grupos, onde vê-se que não houve significância estatística entre os escores.

Fonte: Dados da pesquisa (2019).

\section{Medidas Preventivas}

$\mathrm{Na} \mathrm{EB}$, quando menor o escore, maior o risco para o desenvolvimento da lesão por pressão (LP). O grupo NI evidenciou alto risco na $\mathrm{EB}(12,89 \pm 0,71)$, maior tempo de acamamento $(25,18 \pm 5,81$ anos), maior incidência de comorbidades neuronais $(70 \%)$ e uso de medicações neurológicas $(73,33 \%)$, conduto a incidência de LP foi $0 \%$. As condições da pele e as medidas preventivas adotadas influenciaram essa incidência. O grupo I e AE apresentaram 
na EB risco moderado e menor tempo de acamamento, contudo houve incidência de LP, e isso está relacionado ao fato que esses dois grupos são constituídos por idosos, população mais vulnerável a desenvolver esse tipo de lesão.

As medidas preventivas avaliadas foram: mudança de decúbito, inspeção diária da pele, limpeza do paciente, manutenção do paciente seco, uso de creme barreira, ingesta hídrica, uso de creme hidratante, não realização de massagens de conforto, manutenção da cabeceira do leito a um ângulo não superior a $30^{\circ}$ e superfície de repouso utilizada.

Quanto à mudança de decúbito, 55,56\% do grupo NI conseguiram mudar de posição sem auxílio e 22,22\% tinham a sua posição mudada com frequência. No grupo I, metade da população era mudada de posição, contudo a periodicidade em $66,67 \%$ era superior a duas horas. No grupo AE, $45 \%$ mudavam de posição sem auxílio, $30 \%$ não eram reposicionados e $25 \%$ mudavam de decúbito com auxílio do cuidador, contudo a periodicidade era superior a duas horas na maioria dos casos.

A pressão é a principal causa para a formação de uma LP, pois leva à diminuição ou obliteração do fluxo sanguíneo e consequentemente da oferta de oxigênio e nutrientes, elementos essenciais para a vitalidade dos tecidos (POTTER et al., 2013), com isso a mudança de decúbito é considerada o pilar da prevenção da LP, pois reduz o tempo que determinados pontos se encontram sob pressão (BLANCK; GIANNINI, 2014). O reposicionamento redistribui a pressão e, consequentemente melhora a circulação sanguínea nas áreas isquêmicas, evitando-se assim a deterioração tissular. A frequência de duas horas em uma única posição é o tempo máximo recomendado (BRASIL, 2013).

Em relação à inspeção diária da pele, esse cuidado foi realizado pela maioria dos cuidadores dos três grupos. No grupo NI essa medida preventiva foi realizada em $100 \%$ dos participantes, no grupo I foi realizada em $91,67 \%$ e no grupo AE em 95\%. A inspeção diária da pele deve ser realizada de forma meticulosa para reconhecer alterações da integridade cutânea, propiciando dessa forma uma detecção precoce de lesões. Para uma inspeção adequada, deve-se ter atenção às áreas corporais de maior risco para desenvolvimento de LP, como as regiões: sacral, trocanteriana, occipital, escapular, calcâneo, ísquio e maleolar (BRASIL, 2013).

A manutenção do paciente seco e limpo é essencial para preservação da integridade da pele, visto que a umidade torna a pele mais vulnerável, propiciando o desenvolvimento de lesões (BRASIL, 2013). No grupo NI, 100\% dos participantes era mantidos limpos e $66,67 \%$ secos. O grupo I, a limpeza era realizada em $100 \%$ e a manutenção do paciente seco em $83,33 \%$ e o grupo AE, a limpeza também era realizada em $100 \%$ dos participantes e a manutenção do paciente seco em $95 \%$ dos casos.

A utilização do creme barreira é essencial para minimizar o contato da umidade com a pele (BRASIL, 2013). Este confere proteção contra fluídos corporais, como urina e fezes, evitando assim que a pele fique úmida, e consequentemente mais susceptível à ruptura (CAMPOS et al., 2016). Os cuidadores do grupo NI não faziam uso desse produto, já do grupo I, 8,33\% faziam uso e do grupo AE 15\%.

As condições da pele interferem no aparecimento de uma LP. A pele hidratada e com turgor cutâneo preservado mostra maior resistência aos agentes externos, como pressão, fricção e cisalhamento, minimizando dessa forma sua ruptura (BRASIL, 2013). As condições da pele podem ser melhoradas através de um adequado aporte hídrico e nutricional, além disso, a pele ressecada pode ser atenuada com o uso de cremes hidratantes.

É recomendada a aplicação de hidratante na pele seca ou em áreas ressecadas no mínimo uma vez ao dia (BRASIL, 2013). O grupo NI não fez uso de creme hidratante, já o grupo I e AE apresentaram um índice de uso de 83,33\% e $75 \%$, respectivamente.

$\mathrm{O}$ aporte hídrico adequado também influencia no surgimento da LP. A hidratação adequada, assim como a nutrição é essencial para prevenir e manter a integridade das barreiras cutâneas (CAMPOS et al., 2016). No grupo NI $66,67 \%$ dos participantes apresentaram uma ingesta hídrica adequada. O grupo I apresentou a menor porcentagem em comparação com os demais grupos, correspondendo a $33,33 \%$, e o grupo AE, a ingesta hídrica adequada esteve presente em $55 \%$ dos casos.

As massagens de conforto em áreas de pressão são contraindicadas como medida preventiva de LP, pois pode haver vasos sanguíneos deteriorados ou pele fragilizada, culminando assim na ruptura da pele (BRASIL, 2013). No grupo NI, todos os cuidadores evitavam a realização dessa prática. No grupo I, 91,67\% não realizavam, já o grupo $\mathrm{AE}$ evidenciou maior frequência em relação aos demais grupos, sendo que cerca de $15 \%$ dos participantes recebiam essas massagens.

$\mathrm{O}$ reposicionamento do indivíduo acamado não deve exceder $30^{\circ}$ na posição de semi-Fowler, pois assim evita-se o cisalhamento e o aumento da pressão na região sacral (BRASIL, 2013). Nos três grupos a manutenção da cabeceira do leito não superior a $30^{\circ}$ foi utilizada pela maioria dos cuidadores. O grupo NI e AE evidenciou que $100 \%$ dos participantes eram mantidos em um ângulo de até $30^{\circ}$, já o grupo I, 91,67\% eram mantidos nessa angulação.

A superfície utilizada para repouso também contribui para o desenvolvimento de LP. Os dispositivos especializados para redistribuir a pressão são idealizados para controlar as cargas teciduais e o microclima (umidade e temperatura elevada) (CAMPOS et al., 2016). O grupo NI $66,67 \%$ utilizou a rede como superfície de repouso. O grupo I 75\% utilizava o colchão normal e o grupo AE a superfície mais utilizada foi o colchão normal (45\%), seguida pela rede (35\%), colchão pneumático (15\%) e piramidal (5\%).

As superfícies mais utilizadas para redistribuição de pressão nesse trabalho foram o colchão pneumático e piramidal. Contudo, mesmo com o uso de uma superfície de suporte, não se deve negligenciar a mobilização e o reposicionamento corporal (CAMPOS et al., 2016). A utilização da rede mostrou-se significante (37\%). Segundo Ramos et al. (2016), que realizou um ensaio reflexivo sobre a rede como prevenção de lesão por pressão, traz essa superfície como confortável e mais aerada que o colchão comum, culminando assim na diminuição da temperatura. $\mathrm{O}$ colchão casual aumenta a temperatura e consequentemente há o aumento da transpiração e com isso, aumento da umidade que torna a pele mais susceptível à ruptura.

O processo de avaliação de risco e a aplicação de medidas preventivas são de extrema importância para a manutenção da integridade da pele dos indivíduos restritos 
ao leito. O Protocolo para Prevenção de Úlceras por Pressão traz as medidas preventivas para a prevenção de LP, conforme a classificação de risco na EB (BRASIL, 2013).

Para os indivíduos com risco baixo (15 a 18 pontos) preconiza-se a utilização de cronograma de mudança de decúbito, otimização da mobilidade, proteção da região dos calcanhares, manutenção da umidade, nutrição, fricção e cisalhamento e uso de superfícies de redistribuição de pressão. Para aqueles com risco moderado (13 a 14 pontos) deve-se prosseguir as medidas utilizadas no risco baixo e realizar mudança de decúbito com posicionamento a $30^{\circ}$. No risco alto (10 e 12 pontos) deve-se continuar as intervenções do risco moderado combinadas com a mudança de decúbito frequente e a utilização de coxins para facilitar a lateralização a $30^{\circ}$. E no risco muito alto ( $<9$ pontos) devem-se prevalecer as intervenções realizadas no risco alto, além do controle da dor (BRASIL, 2013).

Todos os indivíduos vulneráveis, independente do grupo etário, devem receber as recomendações para a prevenção da LP. Essas medidas devem ser adotadas por todos os profissionais envolvidos no processo de cuidar, seja em ambiente hospitalar ou domiciliar (BRASIL, 2013).

Em relação às medidas preventivas utilizadas pelos cuidadores, levando em consideração as medidas preconizadas pelo Ministério da Saúde para a classificação de risco segundo a $\mathrm{EB}$, viu-se que estes utilizavam as medidas para baixo e moderado risco, e isso repercutiu positivamente na baixa incidência de LP mostrada neste estudo.

Apesar da Escala de Braden ser um instrumento importante para identificar indivíduos em risco para o desenvolvimento de uma lesão por pressão, a classificação pela escala não mostrou associação com a ocorrência de lesão por pressão. Resultado semelhante foi encontrado em um estudo desenvolvido em unidade de terapia intensiva com indivíduos restritos ao leito (COSTA et al., 2015).

\section{Considerações Finais}

A Escala de Braden não mostrou ser um instrumento eficaz na predição de risco de lesão por pressão no âmbito domiciliar. Apesar de ser um instrumento bastante utilizado pelos profissionais da área da saúde, o presente trabalho demostrou que a alta sensibilidade e especificidade da EB é questionável para aplicar em indivíduos acamados em domicílio.

Além disso, foi demostrado que os cuidados foram essenciais para baixa incidência de LP, diminuído sua associação entre idade e tempo de acamado. Características socioeconômicas também têm suas influências diminuídas na manifestação da LP de acordo com as medidas preventivas adotadas pelos cuidadores.

Perante isso, a predição de risco de lesão por essa escala não pode ser usada como a única ferramenta. A associação ao julgamento clínico do enfermeiro é essencial para se avaliarem outros fatores relacionados que não são abordados nessa ferramenta. Ainda é necessário averiguar se os achados dessa pesquisa dependem de condições regionais e/ou culturais relacionadas.

\section{Referências}

ALVES, C.R.; COSTA, L.M.; BOUÇÃO, D.M. N. Escala de Braden: a importância da avaliação do risco de úlcera de pressão em pacientes em uma unidade de terapia intensiva. Revista Recien, v. 6, n. 17, 2016. Disponível em: https:// www.recien.com.br/index.php/Recien/article/view/147/223. Acesso em: 9 mar. 2019.

BERNARDES, G. M. et al. Perfil de multimorbidade associado à incapacidade entre idosos residentes na Região Metropolitana de Belo Horizonte, Brasil. Ciênc. Saúde Colet. v. 24, n. 5, 2019. Disponível em: https://www.scielo. br/pdf/csc/v24n5/1413-8123-csc-24-05-1853.pdf. Acesso em: 7 dez. 2019.

BLANCK M.; GIANNINI, T. Prevenção de úlcera por pressão. In:_. Úlceras e feridas: as feridas tem alma. Rio de Janeiro: Di Livros, 2014. p. 341-346.

BRASIL. Lei ${ }^{\circ} 10.741$, de $1^{\circ}$ de outubro de 2003. Dispõe sobre o Estatuto do Idoso e dá outras providência. Diário Oficial da União, Brasília, DF, $1^{\circ}$ out. 2003. Disponível em: http://www.planalto.gov.br/ccivil_03/leis/2003/110.741. htm. Acesso em: 25 nov. 2019.

BRASIL. Ministério da Economia. Relação Anual de Informações Sociais (RAIS). 2018. Disponível em: http:// www.rais.gov.br/sitio/download.jsf\#portaria. Acesso: 10 dez. 2019.

BRASIL. Ministério da Saúde. Anexo 02: Protocolo para prevenção de úlcera por pressão. Ministério da Saúde/ Anvisa/Fiocruz. 2013. Disponível em: https://proqualis.net/ sites/proqualis.net/files/000002429jFPtGg.pdf. Acesso em: 22 mar. 2019.

CAMPOS, M. G. C. A. et al. Lesão por pressão. In: VASCONCELOS, J. M. B.; CALARI, M. H. L. Feridas complexas e estomias: aspectos preventivos e manejo clínico. João Pessoa: Ideia, 2016. p. 192-238. Disponível em: http://www.corenpb.gov.br/wp-content/ uploads/2016/11/E-book-coren-final-1.pdf. Acesso em: 12 mar. 2019.

CERVO, A. L.; BERVIAN, P. A.; SILVA, R. Pesquisa: conceitos e definições. In: Metodologia Científica. 6. ed. São Paulo: Pearson Prentice Hall, 2007. p.61.

COREN - DF. Conselho Regional de Enfermagem do Distrito Federal. Muda terminologia para úlcera por pressão. 2016. Disponível em: https://www.coren-df.gov. $\mathrm{br} /$ site/muda-terminologia-para-ulcera-por-pressao/. Acesso em: 9 mar. 2019.

COSTA, A. C. et al. Úlcera por presión: incidência y fatores demográficos, clínicos y nutricionales associados en pacientes de uma unidad de cuidados intensivos. Nutr. Hosp., v. 32, n. 5, 2015. Disponível em: http://www. aulamedica.es/nh/pdf/9646.pdf. Acesso em: 2 dez. 2019. 
DEBON, R. et al. Visão do enfermeiro quanto a aplicação da escala de Braden no paciente idoso. J. res.: fundam. care. online, v. 10, n. 3, 2018. Disponível em: http:// www.seer.unirio.br/index.php/cuidadofundamental/article/ viewFile/6210/pdf_1. Acesso em: 9 mar. 2019.

IBGE - Instituto Brasileiro de Geografia e Estatística. 2017. Disponível em: https://cidades.ibge.gov.br/brasil/ce/aracati/ panorama. Acesso em: 26 nov. 2019.

IBGE - Instituto Brasileiro de Geografia e Estatística. Cadastro Central de Empresas. 2016. Disponível em: https://www.opovo.com.br/jornal/economia/2018/06/cearatem-a-segunda-pior-media-salarial-do-pais.html. Acesso em: 26 nov. 2019.

IBGE - Instituto Brasileiro de Geografia e Estatística. Pesquisa Nacional por Amostra de Domicílios. 2011. Disponível em: http://g1.globo.com/brasil/noticia/2012/09/ maioria-dos-brasileiros-e-solteiro-mas-57-tem-algum-tipode-uniao-conjugal.html. Acesso em: 25 nov. 2019.

IBGE - Instituto Brasileiro de Geografia e Estatística. Pesquisa Nacional por Amostra de Domicílios Contínua: cor ou raça. 2018. Disponível em: https://www.opovo.com. br/noticias/fortaleza/2019/05/22/apenas-5--da-populacaono-ceara-se-reconhece-como-negra--aponta-pnad.html. Acesso em: 25 nov. 2019.

IBGE - Instituto Brasileiro de Geografia e Estatística. Pesquisa Nacional por Amostra de Domicílios Contínua: educação. 2018. Disponível em: https://educa.ibge.gov.br/ jovens/conheca-o-brasil/populacao/18317-educacao.html. Acesso em 25 nov. 2019.

IBGE - Instituto Brasileiro de Geografia e Estatística. Pesquisa Nacional por Amostra de Domicílios Contínua: quantidade de homens e mulheres. 2018. Disponível em: https://educa.ibge.gov.br/jovens/conheca-o-brasil/ populacao/18320-quantidade-de-homens-e-mulheres.html. Acesso: 25 nov. 2019.

IBGE - Instituto Brasileiro de Geografia e Estatística. Tábuas Completas de Mortalidade: expectativa de vida. 2018. Disponível em: https://agenciadenoticias.ibge.gov.br/ agencia-noticias/2012-agencia-de-noticias/noticias/23206expectativa-de-vida-do-brasileiro-sobe-para-76-anosmortalidade-infantil-cai. Acesso em: 25 nov. 2019.

MICHEL, M. H. Metodologia e pesquisa. 2. ed. São Paulo: Atlas, 2009. 37 p.

MORAES, J. T. et al. Riesgo de aparición y prevalencia de lesión por presión en atención primaria. Gerokomos, v. 30, n. 2, 2019. Disponível em: http://scielo.isciii.es/pdf/geroko/ v30n2/1134-928X-geroko-30-02-93.pdf. Acesso em: 28 nov. 2019.

MORO, J. V.; CALARI, M. H. L. Úlcera por pressão após a alta hospitalar e o cuidado em domicílio. Esc. Anna Nery Rev. Enferm., v. 20, n. 3, 2016. Disponível em: https://www.scielo.br/pdf/ean/v20n3/1414-8145ean-20-03-20160058.pdf. Acesso em: 22 fev. 2019.

POTTER, P. A. et al. Integridade da pele e cuidados de enfermagem. In: Fundamentos de Enfermagem. 8. ed. Rio de Janeiro: Elsevier; 2013. p. 1204-1261.

PRANHOS, W. Y.; SANTOS, V. L. Avaliação de risco para úlceras de pressão por meio da escala de Braden, na língua portuguesa. Rev. Esc. Enfem. USP, v. 33, n. Especial, 1999. Disponível em: http:/www.ee.usp.br/reeusp/upload/ pdF/799.pdF. Acesso em: 22 mar. 2019.

RAMOS, R. L. et al. Ensaio reflexivo sobre o uso da rede de dormir como prevenção da úlcera por pressão. Rev. cuba. enferm. v. 32, n. 1, 2016. Disponível em: http:// scielo.sld.cu/pdf/enf/v32n1/enf12116.pdf. Acesso: 29 nov. 2019.

VIEIRA, C. P. B. et al. Ações preventivas em úlceras por pressão realizadas por enfermeiras na atenção básica. J. res.: fundam. care. online, v. 8, n. 2, 2016. Disponível em: http://www.seer.unirio.br/index.php/cuidadofundamental/ article/view/4617/pdf_1897. Acesso em: 9 mar. 2019.

VIEIRA, V. A. S. et al. Risco de lesão por pressão em idosos com comprometimento na realização de atividades da vida diária. Revista de Enfermagem do Centro-Oeste Mineiro, v. 8, 2018. Disponível em: http://seer.ufsj.edu.br/ index.php/recom/article/view/2599/1940. Acesso em: 20 mar. 2019.

Recebido em: 31/12/2019 Aceito em: 10/04/2020 